\title{
Analysis of Foraging Behavior in Ant Swarms Based on StarLogo Simulation
}

\author{
Bailong Liu \\ College of Computer Science and Technology \\ Harbin Engineering University \\ Harbin, HeiLongjiang, 150001, China \\ 1bl624@163.com
}

\author{
Rubo Zhang and Changting Shi \\ College of Computer Science and Technology \\ Harbin Engineering University \\ Harbin, HeiLongjiang, 150001, China \\ \{zrbzrb, shichangting\}@hrbeu.edu.cn
}

\begin{abstract}
Swarm Intelligence which is inspired by social animals has gained a lot of attention recently. It always appeals to the collective behaviors observed in social animals. Foraging behavior in ant colonies have come to be viewed as a prototypical example of how complex group behavior can arise from simple individuals. In this paper, foraging process with two sources observed in ant system is simulated by Starlogo. Three aspects, the distances of sources, the evaporation speed of the pheromone, the number of individuals, are considered respectively. Each individual is controlled by the Finite State machine. The completion time is the estimation standard of system performance. The results of simulations show the power law relationship between the completion time (also collision frequency) and the flux of foragers. The work presented here guides a better understanding on self-organization and Swarm Intelligence. It can be used to design more efficient, adaptive, reliable intelligent systems.
\end{abstract}

Index Terms - Swarm Intelligence, foraging behavior, Starlogo simulation

\section{INTRODUCTION}

In recent years, swarm intelligence (SI) has been paid more attention by researchers in different fields, such as artificial intelligence, biology, society, economy and so on. Inspired by complex collective behavior of social animals such as ants, birds and so on, the swam-based system has the characters of adaptation, robustness, flexibility and reliability. It is believed that the global patterns emerge from the local interactions between the individuals and the environment. The system can search the solutions of complex problems without central control and global information. So the phenomenon of SI can be considered as self-organization. To explain the selforganization in swarm intelligence system, it is useful to research the collective behaviors observed in social animals such as aggregation, foraging, nest building and so on. Ant colonies have come to be viewed widely as a typical example of how complex group behavior can arise from simple individuals [1].

Foraging behavior in ants which is mostly modeled and studied by many researchers occurs in a wide variety of strategies, with several of these strategies exhibiting characteristics of self-organization: emergent pattern formation, multistability and bifurcations. Some species adopt the combination of these strategies. This depends on the environmental conditions such as food distribution[2]. Holldobler[3,4] provides a thorough classification of the foraging strategies in ant colony. This system of organizing the foraging methods breaks all phenomena into three categories: hunting, retrieving, and defense. The first two categories are observed in any ant species mostly, and they lie on the communication by pheromone or contact, which is useful in the process of recruitment. Also Holldobler divides the various types of foraging recruitment into four groups: (1) Tandem Running; (2) Group Recruitment; (3) Trail-based Group Recruitment; (4) Mass Recruitment. The fourth one is one of the most complex forms of social behavior in ant colony. In this paper we will consider the case of mass recruitment. In the process of foraging, the scout returns to the nest and lays a pheromone trail after it discovered a food source. Then the foragers will be activated by the trails and become recruits. These recruits can become recruiters in their turn.

In order to research decentralized systems, researchers are choosing decentralized models for the organizations, technologies and theories that they have construct in the world. In our previous work, Monte Carlo method is used to simulate the stochastic aspects in the system. Here we appeal another tool named StarLogo to visualize and analyze foraging behavior. StarLogo is designed to help researchers develop new ways of thinking about and understanding decentralized systems, as well as self-organization in SI[17]. From StarLogo we can get the effect of the individual interactions in a global view. Although previous works have been done by other researchers, here the distribution of the sources, the evaporation speed of the pheromone and the number of individuals are considered. The results are analyzed based on the three aspects, which make us know the self-organization in SI system more clearly, and help to build more complete system model in future.

The paper is organized as follows: related works on foraging behavior in ants and robots are overviewed in section 2. In section 3, the introduction of Starlogo and the strategy of the foraging behavior are given. Then the simulations and analyses are showed in section 4.

\section{RELATED WORKS}

Inspired by the recruitment process of the ant foraging behavior, most researchers of intelligence applied it to different areas, such as optimization algorithms, robotics. The ant colony algorithm was developed by M.Dorigo, which is also inspired by foraging behavior of ants. It is then well used in Travel Salesman Problems(TSP), Assignment Problems, 
Job-shop and other optimal problems[1]. The simple interactions observed in foraging behavior of ants also inspire many robotic researchers to coordinate the robots. This makes the robotics system higher reliability, adaptability and flexibility. Romas et al. took advantage of the cognitive maps introduced in [5], and endowed the individual life cycle. Through evolution of the individuals, the system can adapt the dynamic environment well. Tsankova and Georgieva[6] simulated a two-robot foraging behavior using artificial pheromone. They concluded that the performance increased as the number of the robot increased. Sugawara[7] defined some basic behavior of robots, and gave the relation between the number of the robot and the completion time of the foraging task in both physical experiments and simulations.

Several models aiming to study the influence of different parameters in foraging behavior have been proposed. Edelstein-Keshet et al. present two models based on trail length, but both models feature continuous trail-laying rather than mass recruitment[8,9]. The authors analyze the models with respect to global adaptability in response to external conditions. Gazi V. et al analyze the stability of the foraging behavior of an ant swarm by the dynamic equations based on potential field method. They proved that the individuals with sense error would move to the goal cohesively by Lyapunov theory[10]. But their model assumes that each individual has the information of all the others. Chen Weidong[16] et al proposed a queue coordination strategy on local sensing in distributed multiple mobile robots system. Through the strategy, the number of collision reduced a lot in the nest and the deadlocks can also be avoided well.

By far, the most rigorous theoretical representation of a recruitment system based on differential equations is developed by Nicolis and Deneubourg[11]. They account for the competition between trails in the presence of an arbitrary number of sources. The model takes a macroscopic approach to the mass recruitment system, using pheromone concentration as the principal variable. It is mainly devoted to the choice of orientation among more than one trail upon leaving the nest. Nicolis and Deneubourg make important initial assumptions regarding the recruitment system, as listed below: (1) Pheromone trails are always followed error-free from nest to food source. (2) Ants are either within the nest or following a trail and wandering does not occur. (3) Direct interactions between individuals are ignored; only stigmergy(through pheromone) is possible. (4) The outgoing flux of ants from the nest is a constant. These link the rules of individual to the mathematical descriptions. But their model in the precondition of the food will never decrease. Also the distance between the source and the nest is not considered.

Except for the theoretical models and real robotics system, a good simulation is also very important for researchers especial the beginners. It can visualize the process of the system with less cost. That is why we use Starlogo simulation here.

\section{SYSTEM DESCRIPTION BASED ON STARLOGO}

\section{A. Introduction of Starlogo}

StarLogo is a programmable modeling environment for exploring the behaviors of decentralized systems, such as bird flocks, traffic jams, and ant colonies. It is designed especially for use by students. By far, From 2-D world of openStarLogo to 3-D world of SrarLogo TNG, there are many versions of StarLogo. They give us a new vision of the complex system.

StarLogo is an extension of the Logo programming language. With traditional versions of Logo, we can create drawings and animations by giving commands to a "turtle" on the computer. StarLogo extends this idea by allowing us to control numbers of turtles in parallel. In addition, StarLogo makes the "turtles" world computationally active. We can also write programs for thousands of "patches" which make up the environment. Turtles and patches can interact with each another. For example, the turtles can be programmed to sense around the world, and change their behaviors based on what they sense in the patches around. StarLogo is particularly well-suited for modeling complex decentralized systems.

StarLogo includes three main types of characters:

1) Turtles. The main inhabitants of the StarLogo world are graphic creatures named "turtles" whose shapes can be defined by users. A turtle can be used to represent almost any type of object: an ant in a colony, a car in a traffic jam, an antibody in an immune system and so on. Each turtle has a position, a heading, a color, and a pen for drawing. It can be added more specialized traits and properties. In StarLogo, the actions and interactions of thousands of turtles can be controlled in parallel.

2) Patches. Patches are pieces of the environment in which the turtles live. Patches are not merely passive objects upon which the turtles act. Like turtles, patches can also execute StarLogo commands, and they can act on turtles and other patches. Patches are arranged in a grid, with each patch corresponding to a square in the grid area.

3) Observer. The observer looks on the turtles and patches from a bird's eye perspective. The observer can create new turtles, and it can monitor the activity of the existing turtles and patches[17].

In this paper, a turtle represents an ant which forages from the nest to the sources. The nest, sources, and pheromone are represented by patches with different colors. The nest colored brown is in the center of the environment. The source is yellow and the pheromone is green. It should be noted that the pheromone patches with higher concentration is brighter than lower ones.

\section{B. Strategy and System settings}

In the Starlogo simulation, the rules of the individuals are also satisfied the mass recruitment in ant system. All the individuals have three basic behaviors: searching, following and going home. Negative feedback in foraging behavior is the evaporation of the pheromone. So the pheromone will decrease in a certain speed. And positive feedback is the pheromone laying in the way to nest. The basic behaviors are explained as follows:

1) Searching: In this process, the individuals will walk randomly from the nest until the food is found. The aim of this behavior is to find the sources. If an individual senses the pheromone in the phrase, it will select the following behavior. 
And if the individual found a source, then it will select going home behavior.

2) Following: In this phrase, the individual will move to the grid with pheromone far away from the nest. Here exists a counter. When the individual moves certain steps in one direction before the food is found, it will change its heading randomly. If the individual is in the nest, it has higher probability to select one trail with higher pheromone at a probability of $P_{i}$.

$$
P_{i}=\frac{\left(1+c_{i}\right)^{2}}{\sum_{j=1}^{s}\left(1+c_{j}\right)^{2}}
$$

$c_{i}$ is the concentration of pheromone on the trail of source $i . s$ is the number of the sources. Here $s=2$ and $i=1,2$.

3) Gong home: When an individual finds the source, it will go home directly. In this process, the individual also releases the pheromone to guide others. If the individual reaches home, it will turn around, and select searching or following behavior.

So the mechanism of behavior selection is controlled by Finite State Machine (FSM) easily. The FSM in foraging behavior is showed in Figure 1.

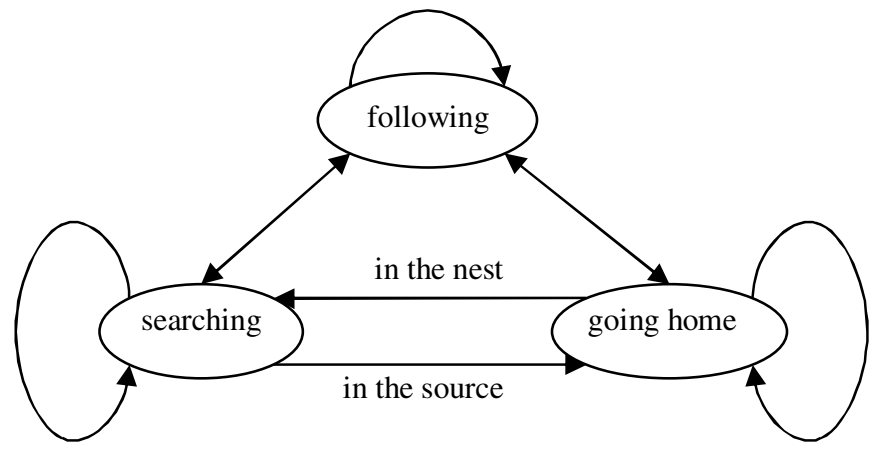

Fig.1 The Finite State Machine in foraging behavior

The environment is a $50 \times 50$ grids plane, as showed in Figure 2. The environment is a bounded field. When the ant reaches to the bound, it turns around and conducts the searching behavior. There are two sources in the whole field. And the nest is in the center of the square. The distance proportion of two sources to nest is $3: 2$. Source 1 is nearer. The individuals are in the nest initially.

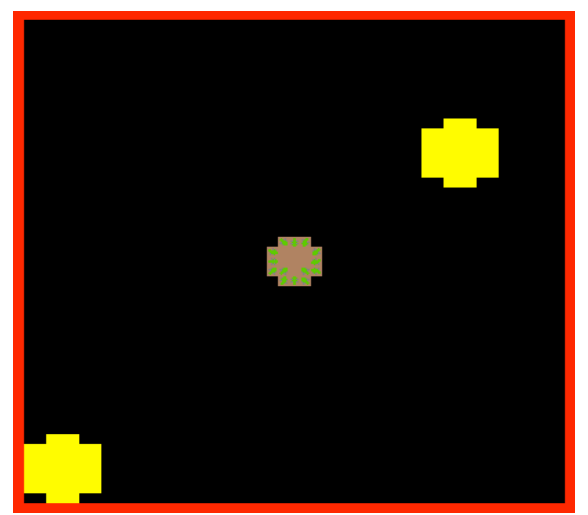

Fig.2 The environment and individuals in Starlogo

\section{SIMULATIONS AND ANALYSES}

In this section, three parameters, the distance of trails, the evaporation of the pheromone, the number of individuals, are analyzed in the simulations. This can make us get more understanding on global patterns emerging from the local interactions, and obtain better control strategies for distributed system.

\section{A. The effect of the trail distance}

In this case, the richness of the food never decreases. We are only interested in the distances of the sources to the nest. Firstly, the distance proportion of two sources and nest is set $1: 1$ (source 1 is moved to the top right corner. There are 37 individuals and the evaporation speed of the pheromone is 22 . The simulation experiments were carried out 20 times. And source 1 is dominantly exploited for 11 times. That is to say two sources have almost the same probability to be exploited. This also satisfies to the theoretical model in [11]. The time evolution of pheromone concentration in one simulation is showed in Figure 3(a). Figure 3(b) shows a screen capture in one simulation when the system is stable. It is seen that most of the individuals exploited one source. Only a few individuals wander and meets source 2 because of the stochastic factor in turning. This keeps the opportunity to find other solutions. But which source will be exploited finally, it depends on which source will be found firstly. It exhibits the multistability of self-organization.

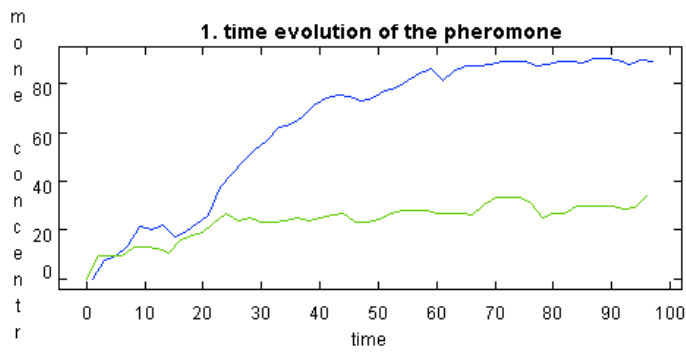

(a) Time evolution of pheromone concetration 


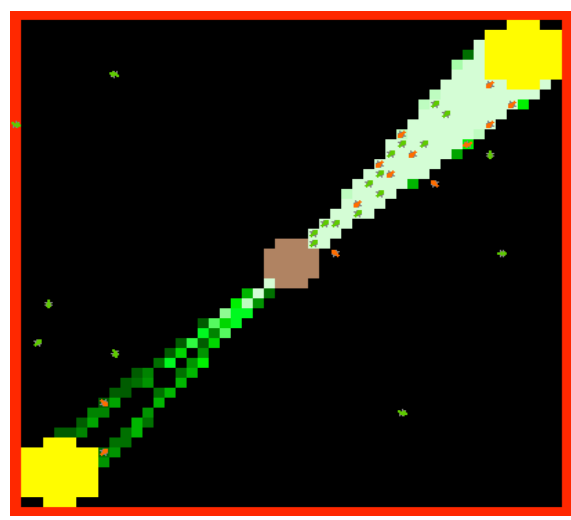

(b) StarLogo simulation

Fig.3 Pheromone distribution in different trails and Starlogo simulation in 1:1 trails

When source 1 is moved to the position whose distance is $2 / 3$ times of source 2 to the nest. Also the experiments are run 20 times. Almost all of the individuals exploited source 1 finally. Only some individuals move randomly out the trails between the nest and the sources. The time evolution of pheromone concentration on both trails in one simulation is showed in Figure 4(a). It is concluded that nearer source has higher probability to be found, so the pheromone concentration on trail 1 is much than trail 2 at first. And due to the enhancement of positive feedback, more and more individuals select trail 1 . We can see that optimal solution of the system will be obtained through the interactions between individuals and environment.

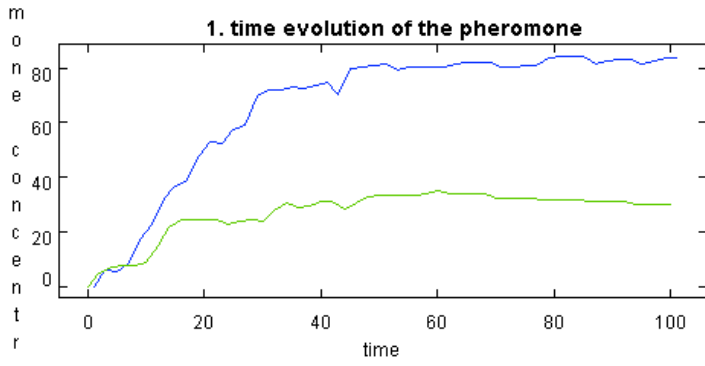

(a) Time evolution of pheromone concentration

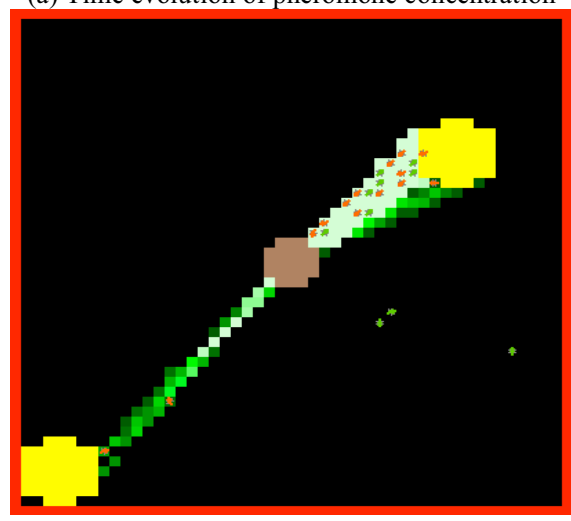

(b) Starlogo simulation

Fig.4 Pheromone distribution in different trails and Starlogo simulation in 2:3 trails

B. The effect of the evaporation speed

The evaporation speed of the pheromone is a negative feedback in the system. In the simulations (distance 2:3), along with the evaporation speed increases, the difference between the pheromone concentration of two trails is much bigger (Figure 5(a)). And finally the pheromone in the longer trail disappears (Figure 5(b)). In the simulations, the evaporation speed $v=59$. When the evaporation speed decrease, the difference between the pheromone concentration of two trails becomes smaller. The difference will decrease to zero when the evaporation speed of pheromone is zero. It can be concluded that larger negative feedback can stand out the optimal solution of the system, while smaller negative feedback is useful to find more solutions of the system.

In real world, the source will be exhausted while time runs. When the richness of the sources can be reduced, the situation is different. The nearer source which is more likely to be found is consumed very fast. But along with the evaporation speed increases, the last source will be exploited as it was met randomly. The food of source 2 decreases slowly. Figure 6(a) shows the time evolution of the food remaining until step 55 at the evaporation speed of 59. In the low value of $v$, the pheromone in trail 2 reduces slowly. If source 1 has been exhausted, there is still enough pheromone in trail 2 to guide free ant to source 2. So the food in farther source then reduces more rapidly than that of higher $v$ (Figure 6(c), (d)).

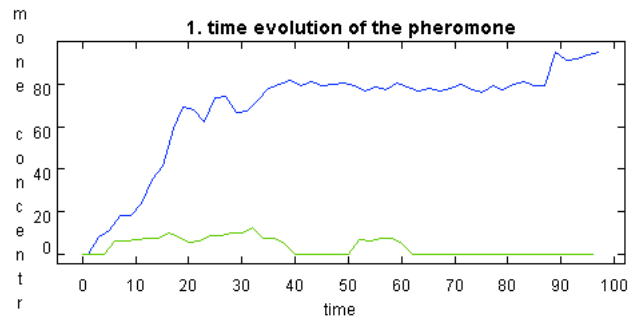

(a) Time evolution of pheromone concentration

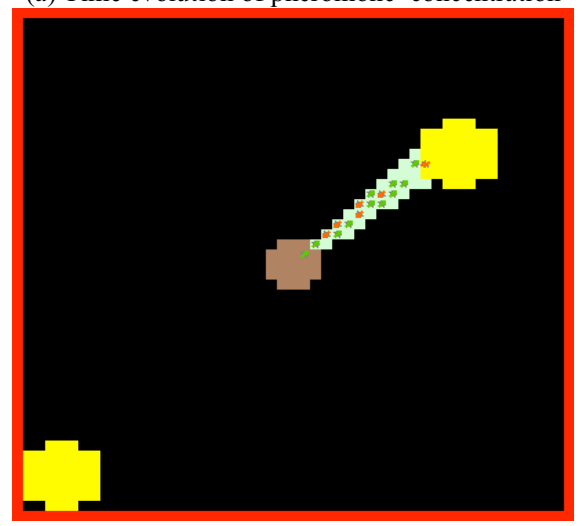

(b) Starlogo simulation

Fig.5 Pheromone distribution in different trails and Starlogo simulation in 2:3 trails with high evaporation speed 


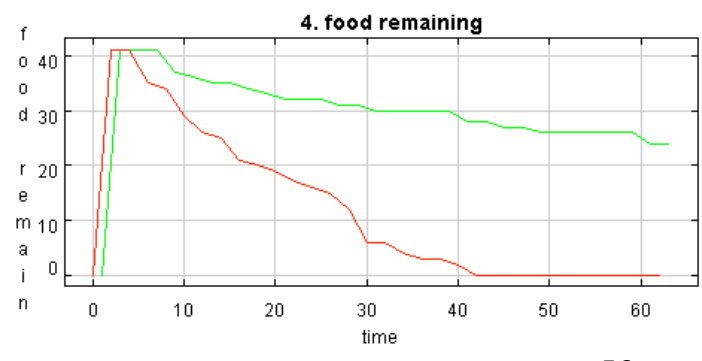

(a) Time evolution of food remaining $(v=59)$

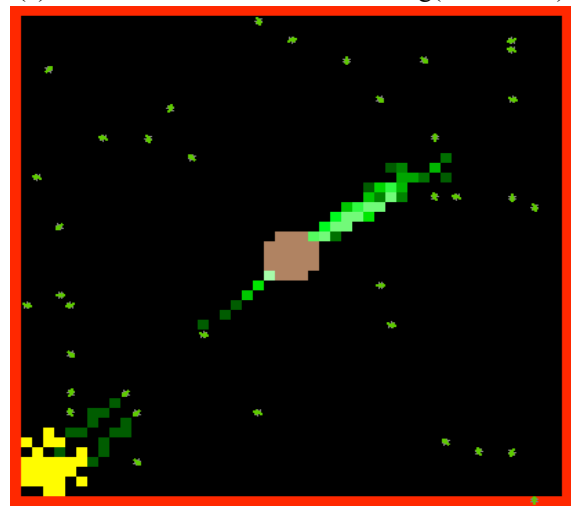

(b) Starlogo simulation $(t=55, v=59$ )

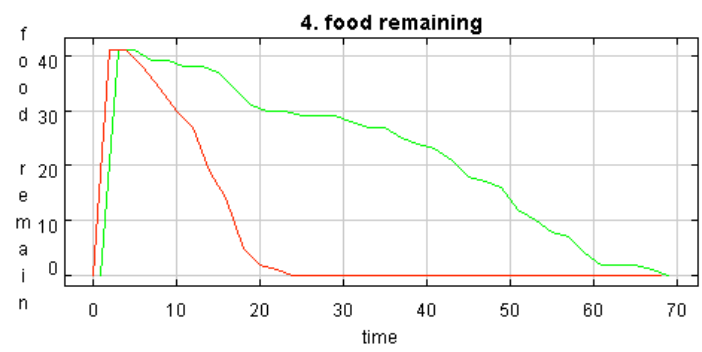

(c) Time evolution of food remaining $(v=22)$

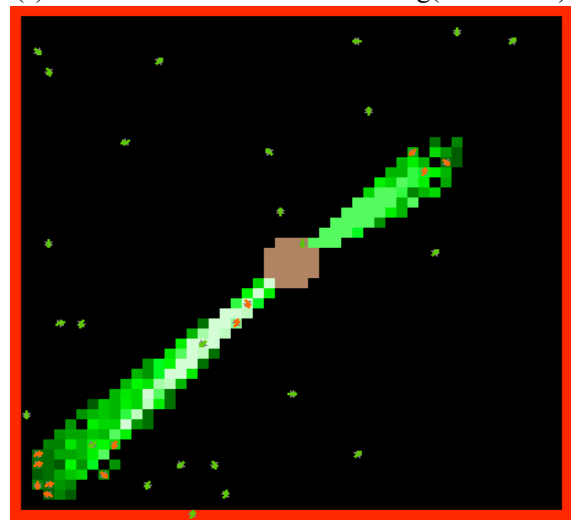

(d) Starlogo simulation( $t=55, v=22$ )

Fig.6 Food remaining in different sources and Starlogo simulation in 2:3 trails with different evaporation speed

\section{The effect of the intensity of individuals}

The number of the individuals is always related to the system performance. And the completion time and the collision frequency represent the performance of the system in certain degree. In the simulation, the completion time means the time between the start and all the food is collected. The collision frequency is the average number of collisions in a unit step. The evaporation speed of pheromone is set 22 . In different number of individuals, the average completion time and collision frequency of 20 simulations is showed in Figure 7. The power law fit is also given in each picture. Both of the curves in Figure 7 show the power law relationship between the two aspects and the intensity of individuals. This means that the interactions between individuals increase exponentially when the number of individuals increases. But the collision occurs more frequently. More interactions apply the difference of the solutions more rapidly by the positive feedback of the system. From (1), the positive feedback is a non-linear term. So the enhancement of it will present a exponential relationship.

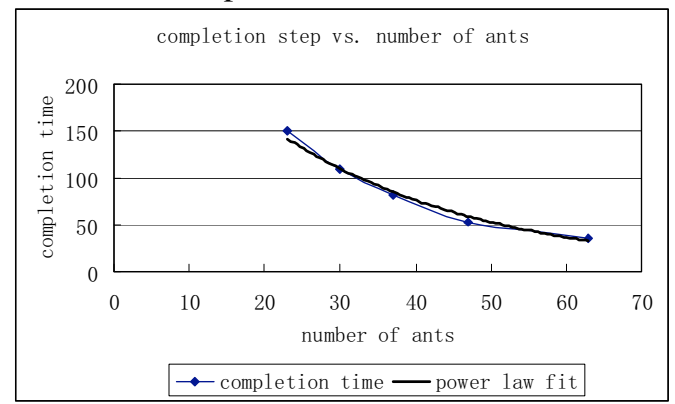

(a) Completion time with different swarm size

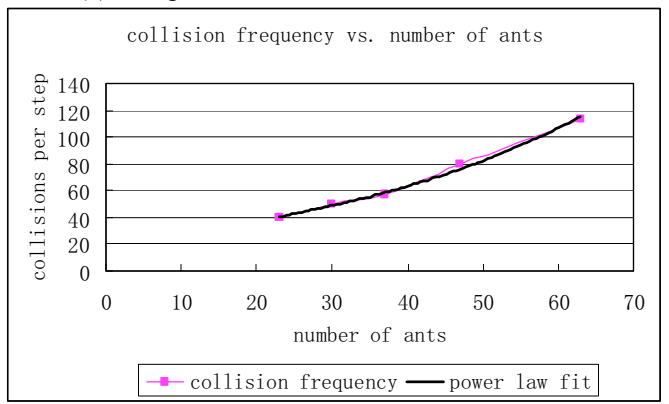

(b)Collision frequency with different swarm size

Fig.7 The completion time and collision frequency in different number of individuals

\section{CONCLUSIONS}

In this paper, the foraging behavior with mass recruitment is simulated in Starlogo. StarLogo is a programmable modelling environment for exploring the behaviors of decentralized systems. The behaviors of system are discussed in three aspects, which are showed in the model of [11]. From the discussion, we can see that nearer source which will be found more probably is exploited first and faster. This depends on the positive feedback of the system. But in the identical case, the multistability occurs. This provides different choices for one solution, and enhances the adaptability of the system. With higher evaporation speed of pheromone, optimal solutions of the system still stable while other ones will be ignored by negative feedback of the system. Finally, the intensity of the individuals has power law relationship with both completion time and collision frequency. In order to have more efficient control on real robots, it is necessary to design strategies to reduce collisions among robots.

The complex swarm-based system emerges from the interaction between simple individuals. Also the self- 
organization comes from the balance between the positive and negative feedback. In future, how the two feedbacks interact with each other and the mechanism of enhancing the useful interactions of individuals will be investigated.

\section{REFERENCES}

[1] Bonabeau E, Dorigo M, Theraulaz G, Swarm intelligence: From natural to artificial systems, first ed., New York Oxford, Oxford University Press, 1999.

[2] Pratt, S. C., Recruiment and other communication behavior in the Ponerine ant Ectatomma ruidum, Ethology. 81 (1989): pp.313-331.

[3] Mark Russell Edelen, Swarm Intelligence and Stigmergy: Robotic implementation of foraging behavior, Graduate School of the University of Maryland, 2003.

[4] Holldobler, B. and Wilson, E. O., The Ants, Cambridge, Harvard University Press, 1990.

[5] Ramos V., Ferandes C., Rosa A.. Social cognitive maps, swarm collective perception and distributed search on dynamic landscapes. Brains, Minds and Media-Journal of New Media in Neural an Cognitive Science, 2005.

[6] Tsankova D. D., Georgieva V. S., From Local Actions to Global Tasks: Simulation of Stigmergy Based Foraging Behavior, IEEE International Conference on Intelligence Systems. (2004): pp.353-358.

[7] Sugawara K., Watanabe T., A study on foraging behavior of simple multirobot system, IEEE 2002 28th Annual Conference of the Industrial Electronics Society( IECON 02), (2002): pp.3085-3090.
[9] Edelstein-Keshet, L., Simple models for trail-following behavior: Trunk trails versus individual foragers, Journal of Mathematical Biology. 32 (1994): pp.303-328.

[10] Gazi V., Passino K. M., Stability analysis of social foraging swarms, IEEE Transactions on Systems, Man, and Cybernetics. 34(2004): pp.539-556.

[11] Nicolis, S.C., Deneubourg, J.L., Emerging patterns and food recruitment in ants: an analytical study, Journal of Theoretical Biolog. 198 (1999): pp.575-592.

[12] Beckers, R., J. L. Deneubourg, S. Goss, Modulation of trail laying in the ant Lasius niger (Hymenoptera: Formicidae) and its role in the collective selection of a food source, Insect Behavior. 6 (1993): pp.751-759.

[13] Nicolis S.C. DETRAIN C., DEMOLIN D., Deneubourg J.L., Optimality of collective choices: A stochastic approach, Bulletin of Mathematical Biology. 65 (2003): pp.795-808.

[14] K. Sugawara, M. Sano, I. Yoshiharat, K. Abet, T. Watanabe, Foraging behavior of multi-robot system and emergence of Swarm Intelligence, IEEE International Conference on Systems, Man, and Cybernetics. (1999): pp.257-262.

[15] G. Beni, J. Wang, Distributed robotic systems and Swarm Intelligence, Proceedings of IEEE Int. Conference on Robotics and Automation. (1991): pp.1914.

[16] Wang Shen, Chen Weidong. Queue coordination strategy based on local sensing in distributed multiple mobile robot system. ROBOT. 2002.6(24): pp.540-544

[17] http://education.mit.edu/starlogo/
[8] Edelstein-Keshet, L., Watmough, J., Ermentrout, G., Trail following in ants: individual properties determine population behavior, Behavioral Ecology and Sociobiology. 36 (1995): pp.119-133. 